\title{
Clinical Reasoning Exercises in Clinical Reasoning: Take a Time-Out and Reflect
}

\author{
Ankit Agrawal, $M D^{7}$, Carlie Stein, $M D^{1,3}$, Dan Hunt, $M D^{2}$, Martin Rodriguez, $M D^{3}$, \\ Lisa L. Willett, MD, MACM ${ }^{1,3}$, and Carlos Estrada, $M D, M S^{3,4}$ \\ ${ }^{1}$ Tinsley Harrison Internal Medicine Residency Program, University of Alabama at Birmingham, Birmingham, AL, USA; ${ }^{2}$ Emory University, Atlanta, GA, \\ USA; ${ }^{3}$ University of Alabama at Birmingham, Birmingham, AL, USA; ${ }^{4}$ Birmingham Veterans Affairs Medical Center, Birmingham, AL, USA.
}

KEY WORDS: clinical reasoning; anchoring; cognitive biases; problem solving; paraneoplastic polyneuropathy.

J Gen Intern Med 33(3):388-92

DOI: $10.1007 / \mathrm{s} 11606-017-4261-1$

(c) Society of General Internal Medicine 2018

In this series, a clinician extemporaneously discusses the diagnostic approach (regular text) to sequentially presented clinical information (bold). Additional commentary on the diagnostic reasoning process (italics) is integrated throughout the discussion.

A 57-year-old male smoker with no significant medical history was admitted to the neurology service with a 3week history of bilateral lower extremity numbness, burning pain, and ataxia.

The description suggests a subacute (less than 1 month) onset of peripheral neuropathy, although the nature of bilateral lower extremity symptoms may also refer to spinal cord or parasagittal region pathology. Many peripheral neuropathies result in both sensory and motor deficits, so additional history and examination will help reveal whether he is weak. Ataxia can also be due to cerebellar, cortical, and basal ganglia pathology, so further exploration of ataxia will need to relate this finding to peripheral neuropathy. The first goal in evaluation is to further characterize the neurologic deficits in order to narrow a broad differential diagnosis.

An early step in the process of clinical reasoning is problem representation, the creation of a brief mental summary highlighting the key points of the case. ${ }^{1}$ This allows the physician to process the information received to create a focused differential diagnosis. Problem representation uses semantic modifiers, descriptive characteristics that define a clinical

Received January 26, 2017

Revised May 15, 2017

Accepted December 4, 2017

Published online January 4, 2018 presentation in general terms. These can include modifiable and non-modifiable risk factors, time course, and presenting symptoms. Examples of semantic modifiers are young versus elderly or acute versus chronic. Other key pieces of information may also be included, such as history of smoking, since it may help consider illnesses that are more common in smokers. The symptoms can be categorized based on the description; in this case the numbness and pain are consistent with sensory neuropathy, but the clinician has not heard of any motor problems yet. Before adding another semantic modifier, the clinician asks for more information. Additional data can further categorize the neuropathy as sensory, motor, or sensorimotor, and thus increase the specificity of the problem representation. Generating a problem representation will enable the clinician to develop initial hypotheses and compare them to illness scripts from previously acquired knowledge and experience. Illness scripts, which constitute a mental summary of a disease, include predisposing conditions, pathophysiology, symptoms, physical exam findings, and other important information. The clinician compares the hypothesis with the illness scripts to see if they fit, may ask for additional information, or may add possibilities in the differential diagnosis. In this case, the clinician recognizes that ataxia may be a symptom of peripheral neuropathy, but more information is needed to evaluate additional possibilities.

The numbness and burning pain originated in the patient's feet and progressively ascended to his abdomen, mid-chest, and upper limbs. He gradually developed difficulty walking and complained of falling to the right side. He denied preceding viral illness, vision changes, bowel or bladder incontinence, or weakness. He denied fever, weight loss, night sweats, cough, shortness of breath, chest pain, vomiting, diarrhea, or rashes. He was previously healthy, did not take any medications or supplements, and had no allergies. He smoked one pack of cigarettes daily for 30 years, drank two beers daily, and occasionally used marijuana. He was monogamous with his wife. His parents and children were healthy.

The additional details suggest a sensory deficit that is symmetric and ascended from the distal lower extremities to the trunk and upper extremities over a 3-week period. Falling to the right side is not explained by sensory deficits alone, and 
suggests that the report of "no weakness" may not be accurate. Ascending neurologic deficits raise the possibility of a form of Guillain-Barré syndrome (GBS), although weakness is the most common presenting symptom with this disorder, and presentation with mainly sensory deficits would be unusual.

Predominantly sensory neuropathies include those caused by diabetes, infections (HIV, leprosy), toxins, medications, vitamin deficiencies (thiamine, B12, vitamin E), vasculitis, monoclonal gammopathies, brain lesions (lacunar infarcts, thalamic lesions, syringomyelia), and paraneoplastic syndromes. Diabetic peripheral neuropathy does not advance this quickly. The history does not suggest significant risk for nutritional deficiency or vasculitis. Smoking places him at risk for malignancy, although he has not experienced weight loss or new pulmonary symptoms. A careful systemic and neurologic examination will confirm the neurologic symptoms and help narrow the differential diagnosis.

The additional information helps the clinician develop a more specific differential diagnosis. Based on the symptoms described, the clinician characterizes the patient's symptoms as predominately sensory neuropathy, rapidly progressing, and acknowledges the history of smoking, which is a risk factor for malignancy. Time course is important in helping to narrow the differential diagnosis. A careful neurologic examination will allow confirmation of the patient's history and better localize the anatomical areas involved. Using this information, the clinician generates initial hypotheses and suggests additional tests for further evaluation.

The patient's temperature was $36.9^{\circ} \mathrm{C}$, heart rate 77 beats per minute, respiratory rate 20 breaths per minute, and blood pressure $161 / 92 \mathrm{~mm} / \mathrm{Hg}$. He appeared thin, well-nourished, with a normal mental status. He exhibited broad-based gait with positive Romberg test. His cerebellar exam was normal. Pronator drift, jaw jerk reflex, and finger taps were not performed. He had decreased light touch and pinprick sensation in the lower extremities extending to the chest, hands, and forearms. Facial sensation was intact. His motor strength was 5/5 throughout. He had absent bilateral Achilles, patellar, and biceps reflexes. Cranial nerve exam was normal. The rest of the physical exam was normal.

The positive Romberg test indicates either posterior column disease or peripheral sensory neuropathy. The sensory findings on exam are consistent with an extensive peripheral neuropathy that involves all extremities and the trunk. His strength is normal, which suggests falling to one side and ataxia are due to sensory deficits alone, and argues against GBS, although the ascending nature of the deficits remains suggestive. Miller-Fisher syndrome, a variant of GBS, presents with acute ataxia, areflexia, and ophthalmoparesis. He is manifesting two of the three features, but the absence of cranial nerve findings argues against this. The absent reflexes are consistent with disruption of the reflex arc, presumably the afferent limb due to acute proprioceptive loss.

The neurologic lesion appears to involve peripheral sensory nerves while sparing motor function, suggesting that imaging of the brain and spinal cord are unlikely to lead to a diagnosis. Because GBS is a consideration, albeit with atypical features, it is reasonable to perform a lumbar puncture (LP) and also obtain electromyography (EMG) and nerve conduction studies (NCS) to further characterize the neuropathy.

The clinician continues to test the hypotheses against the illness scripts for the proposed etiologies. The exam confirms a sensory-predominant neuropathy. Through processing of the history and exam, the clinician narrows the differential and points out that imaging of the brain and spinal cord is unlikely to be useful in identifying a correct diagnosis, but emphasizes the importance of expedited LP and EMG/NCS to rule out a diagnosis of GBS. It is important to recognize that while the clinician starts to prioritize testing and narrow the differential, he or she recognizes that atypical presentations of specific illnesses may not be consistent with the "classic" manifestations of his or her illness scripts, and does not exclude those possibilities. The clinician suggests further tests to support the proposed diagnoses and to look for clues of other diseases.

On laboratory testing, serum sodium level was $130 \mathrm{mM}$ / L. The hemoglobin A1c, vitamin B12, thiamine, TSH, complete blood count, and metabolic panel were all within normal limits. The urine specific gravity was 1.009 . Cerebrospinal fluid (CSF) analysis showed glucose of $63 \mathrm{mg} /$ dL, protein $198 \mathrm{mg} / \mathrm{dL}$, white blood cell (WBC) count 12 cells/cm with $80 \%$ lymphocytes, and 0 red blood cells (RBCs). CSF culture was negative. Brain and spine MRI were normal. Nerve conduction studies (NCS) and electromyogram (EMG) revealed pure sensory loss without motor involvement. Findings were formally reported as "consistent with GBS in the acute phase or a pure sensory neuropathy," but did not mention findings of demyelination.

The laboratory studies exclude a number of conditions that could explain the acute peripheral sensory neuropathy. The mild hyponatremia may or may not be related. This could be explained by syndrome of inappropriate antidiuretic hormone (SIADH) in a patient who is euvolemic based on history and examination; however, the low specific gravity argues against this, and further investigation would be needed. Obtaining serum and urine osmolality is a next step.

An elevated CSF protein with low CSF WBC count $(\leq 5$ $\mathrm{WBC} / \mathrm{mL}$ ) is termed albuminocytologic dissociation and is classically seen in GBS. This patient's CSF protein is elevated but the WBC is not normal. A small percentage of patients 
with GBS have slightly elevated CSF WBC counts, although the finding of $12 \mathrm{WBC} / \mathrm{mL}$ might raise the possibility of a chronic infectious process or a noninfectious inflammatory process.

The EMG/NCS reports "findings consistent with early GBS or a pure sensory neuropathy." The ascending neurologic deficits, elevated CSF protein relative to cell count, neurophysiologic studies, and absence of an alternative explanation should reasonably lead to treatment for GBS with plasma exchange and IVIG given risks of harm to patient with GBS progression outweigh the risks of treatment. Failure to respond to treatment should lead to further investigation, given the lack of motor involvement and no mention of demyelination on NCS.

The clinician processes the results and pays particular attention to the aspects of the exam and testing that support a diagnosis of GBS, with a plan to start treatment. He or she also points out the features of the case-hyponatremia, elevated WBC, lack of demyelination - that may not fit the typical presentation for GBS, suggesting further investigation if the patient does not respond as expected. Recognition of atypical features is important in the process of clinical reasoning, and clinicians should consider slowing down ${ }^{2}$ during the cognitive process, transitioning from an intuitive to analytical reasoning approach, and considering additional diagnostic possibilities. Atypical presentations of common diseases are always possible and occasionally seen in practice; however, clinicians should keep an open mind to avoid common cognitive errors. Many tools designed to avoid errors have been proposed, including the use of reflective reasoning. Groopman and Hartzband have suggested guided reflection using three simple questions when evaluating a diagnostic hypothesis: What else could this be? Is there something that does not fit? Is there more than one diagnosis? ${ }^{3}$ These questions help the clinician consider alternative diagnoses and perform further workup prior to anchoring on a diagnosis of atypical GBS.

The patient was suspected of having sensorypredominant GBS and received intravenous immunoglobulin (IVIG). After 5 days of therapy, his symptoms progressed. He could no longer walk without assistance and developed urinary retention. Deemed a non-response, plasma exchange was initiated. During treatment, his serum sodium levels continued to decrease to $124 \mathrm{mM} / \mathrm{L}$, prompting further laboratory evaluation, with serum osmolality $254 \mathrm{mOsm} / \mathrm{kg}$, urine osmolality $465 \mathrm{mOsm} / \mathrm{kg}$, and urine sodium $61 \mathrm{mM} / \mathrm{L}$. After hospitalization for 9 days, the patient was diagnosed with SIADH and was started on a fluid-restricted diet with oral salt tablets.

Generally, the cause of SIADH falls into four major categories: medications, pulmonary processes, neoplasms, and central nervous system processes. GBS has been associated with SIADH; however, we should consider other causes of SIADH.

Aspects of the presentation that are not consistent with GBS include the pure sensory deficit, the WBCs in the CSF, no demyelination noted on EMG, the progression of illness despite therapy, and worsening hyponatremia. We should stop and reframe the situation. The patient is 57 years old, with significant a smoking history, rapidly progressive sensory neuropathy, and SIADH. Does he have an underlying malignancy causing a paraneoplastic sensory neuropathy and persistent SIADH? We should review his chest X-ray given his smoking history and, if unrevealing, consider a chest $\mathrm{CT}$.

With treatment failure, and identifying a new diagnosis, SIADH, the clinician takes a "diagnostic time-out" and reviews the case presentation and data. The clinician highlights "things that do not fit" or support the current diagnostic hypothesis to determine "what else could this be?" using the guided reflection questions described by Groopman. ${ }^{3}$ This triggers him to reframe the problem representation as: A middle-aged man, smoker, with sub-acute sensory neuropathy, SIADH, mild pleocytosis and elevated CSF protein. This new problem representation is reviewed against additional illness scripts to suggest a new diagnosis and additional studies. Through reflection, the clinician continues to search for a unifying diagnosis.

Further workup for the underlying etiology of SIADH was pursued. A chest X-ray showed a small number of tiny calcified right upper lung nodules without a mass. Chest $C T$ revealed a 4-mm endobronchial lesion in the right middle lobe, mild right hilar lymphadenopathy, and scattered tiny non-calcified nodules (Fig. 1). Transbronchial biopsy of multiple hilar lymph nodes showed small cell lung carcinoma in one node. A CT of the abdomen/pelvis did not show metastases. The patient was diagnosed with limitedstage small cell carcinoma of the lung 14 days into his hospitalization. Testing for serum paraneoplastic antibodies demonstrated anti-Hu (ANNA-1) antibodies with a titer of $1: 15,360$. The patient underwent a first cycle of chemotherapy with carboplatin and etoposide, with improvement in his neuropathy.

\section{DISCUSSION}

The diagnostic process is a complex activity requiring information gathering and clinical reasoning to determine a patient's underlying diagnosis and promptly initiate treatment. This process involves many layers, including history-taking, physical exam, diagnostic testing, and physician consultation. Within this context, estimates suggest that diagnostic error contributes to $40,000-80,000$ deaths in US hospitals each 


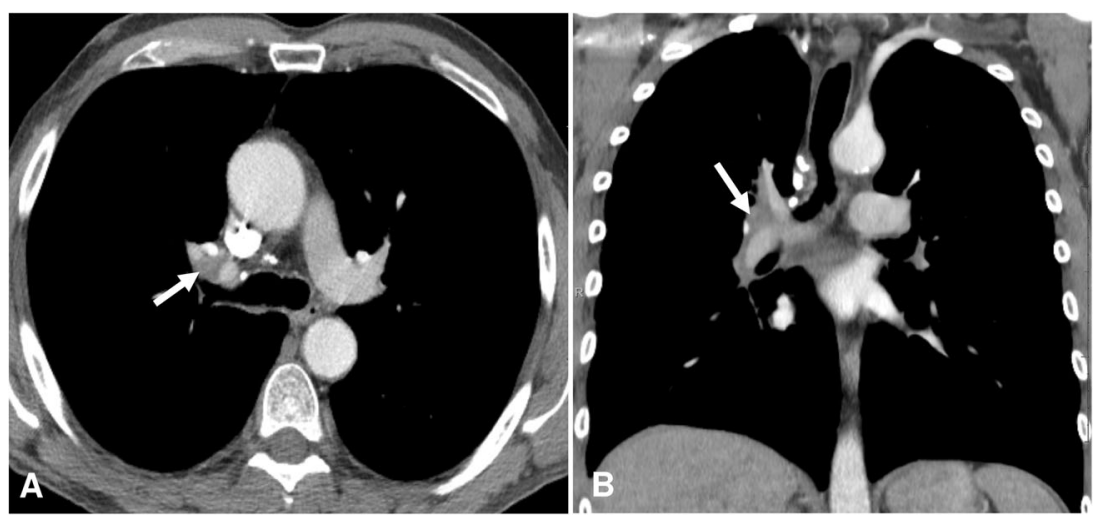

Fig. 1 Computed tomography of the chest showing a 4-mm endobronchial lesion (a) and hilar lymphadenopathy (b).

year. $^{4}$ The majority of these errors are due to cognitive pitfalls rather than deficits in clinician knowledge. This case illustrates how a physician can avoid cognitive biases such as premature closure, anchoring, and diagnostic momentum through reflection.

In this patient with progressive neuropathy, there was room for diagnostic error due to the complex patient presentation and pressure to obtain a quick diagnosis and initiate treatment. While the patient is undergoing treatment, the clinician continues to use careful reflection to keep the door open to further diagnostic workup and differential diagnoses. The clinician continues to reflect on pieces that do not fit typical presentation, and expand as new information comes to hand. While this highlights the importance of clinical reasoning and reflection, the final diagnosis in this patient is delayed, and the patient undergoes several potentially unnecessary treatments over the course of weeks, with a delay in initiating chemotherapy.

In recent studies, important tasks identified during clinical encounters have included framing the problem, diagnosis, management, and reflection. ${ }^{5,6}$ However, physicians generally use intuitive reasoning and omit reflective reasoning in their routine mode of thought. ${ }^{7}$ As the physician processes the initial history and exam, he or she begins to form a problem representation and an initial diagnosis and plan for further workup and treatment. Reflection increases diagnostic accuracy, especially in complex cases, and a systematic approach is essential for maximizing its benefits. ${ }^{7}$ During this phase of the clinical encounter, the physician reviews supporting data for the proposed hypothesis and the three proposed questions: "What else could this be? Is there something that does not fit? Is there more than one diagnosis?" 3 Through these questions, data that may have been overlooked or misinterpreted are considered. Physicians will slow down and use analytical reasoning to interpret the data and create a more refined problem representation and more accurate differential diagnosis. The use of guided reflection will help physicians slow the clinical reasoning process, thus preventing delayed or missed diagnosis.

\section{CLINICAL TEACHING POINTS}

1. Guillain-Barré syndrome (GBS) is characterized by progressive symmetric weakness and hyporeflexia, which can be further classified by the distribution of neurologic involvement. ${ }^{8}$ On EMG/NCS there is typically evidence of demyelinating neuropathy.

2. Pure sensory neuropathy is a rare variant of GBS with diagnostic criteria including "acute-onset symmetric loss, peak deficit within 4 weeks, hyporeflexia, normal motor strength, nerve conduction evidence of demyelination in at least two nerves, monophasic course, no other known cause for neuropathy, no family history of neuropathy."9

3. The differential diagnosis for acquired subacute sensory neuropathies is limited to only a few disease categories: toxicity (pyridoxine, platinum-based chemotherapy), infection (primarily HIV), autoimmune disorders (Sjögren's syndrome, rheumatoid arthritis, systemic lupus erythematosus, autoimmune hepatitis), and paraneoplastic syndromes. Multiple cancer types are associated with paraneoplastic neuropathies, including small cell lung cancer, bronchial carcinoma, breast and ovarian cancer, neuroendocrine tumors, lymphoma, and sarcoma. ${ }^{10}$

4. Paraneoplastic processes must be considered in patients with a strong history of smoking. The presence of anti-Hu antibodies should prompt investigation for a neoplasm, as it is very specific (>99\%) and moderately sensitive (82\%) for underlying malignancy. ${ }^{11}$ Anti-Hu neuropathy typically manifests as a subacute sensory neuropathy with ataxia, decreased or absent reflexes, and CSF pleocytosis.

\section{Acknowledgments:}

Prior Presentations: This case was presented at a clinical problem solving presented as an oral presentation at the 2016 Southern Society of General Internal Medicine meeting, February 18-20, 2016, Atlanta, GA. The clinical information and case discussion closely reflect the topics discussed.

Corresponding Author: Carlie Stein, MD; Tinsley Harrison Internal Medicine Residency Program, University of Alabama at Birmingham, 720 Faculty Office Tower, 510 20th Street South, Birmingham, AL 35294-3407, USA (e-mail: chstein@uabmc.edu). 


\section{Compliance with Ethical Standards:}

Conflict of Interest: The authors declare that they do not have a conflict of interest.

Disclaimer: The opinions expressed in this article are those of the authors alone, and do not necessarily reflect the views of the Department of Veterans Affairs.

\section{REFERENCES}

1. Bowen JL. Educational strategies to promote clinical diagnostic reasoning. N Engl J Med. 2006;355:2217-25.

2. Moulton CA, Regehr G, Mylopoulos M, MacRae HM. Slowing down when you should: a new model of expert judgment. Acad Med. 2007;82(10 Suppl):S109-16.

3. Groopman J, Hartzband P. Thinking about our thinking as physicians. ACP Internist. 2011. Available at: http://www.acpinternist.org/archives/ 2011/10/mindful.htm. Accessed August 16, 2017.
4. Newman-Toker DE, Provonost PJ. Diagnostic errors - the next frontier for patient safety. JAMA. 2009;301(10):1060-2.

5. Goldszmidt M, Minda JP, Bordage G. Developing a unified list of physicians reasoning tasks during clinical encounters. Acad Med. 2013;88(3):390-7.

6. McBee E, Ratcliffe T, Goldszmidt M, et al. Clinical reasoning tasks and resident physicians: what do they reason about? Acad Med. 2016;91(7): 1022-8.

7. Mamede S, Schmidt HG. Reflection in medical diagnosis: a literature review. Health Prof Educ. 2017;3(1):15-25.

8. Van den Berg B, Walgaard C, Drenthen J, Fokke C, Jacobs BC, Van Doorn PA. Guillain-Barré syndrome: pathogenesis, treatment and prognosis. Nat Rev Neurol. 2014;10(8):469-82.

9. Oh SJ, LaGanke C, Claussen GC. Sensory Guillain-Barré syndrome. Neurology. 2001;1:82-6.

10. Sheikh SI, Amato AA. The dorsal root ganglion under attack: the acquired sensory ganglionopathies. Pract Neurol. 2010;10(6):326-34.

11. Molinuevo JL, Graus F, Serrano C, Rene R, Guerrero A, Illa I. Utility of anti-Hu antibodies in the diagnosis of paraneoplastic sensory neuropathy. Ann Neurol. 1998;44(6):976-80. 